\title{
Chemotherapy for a ventilator-supported patient with small cell lung cancer: A case report
}

\author{
RUN XIANG ${ }^{*}$, TIANPENG XIE* and QIANG LI \\ Department of Chest Surgery, Sichuan Cancer Hospital, Chengdu, Sichuan 610041, P.R. China
}

Received March 30, 2015; Accepted May 12, 2016

DOI: $10.3892 / 01.2016 .4861$

\begin{abstract}
Small cell lung cancer (SCLC) is common in thoracic neoplasms, with a high degree of malignancy and rapid tumor progression. SCLC is often diagnosed with widespread metastases at the time of the initial diagnosis. A small proportion of late-stage SCLC patients are in a poor physical condition and exhibit disqualifying chemoradiotherapy indications. The present study reports the case of a patient who presented with lumbago and backache. Following physical examination, computed tomography, bronchoscopy and biopsy, the patient was diagnosed with SCLC with an Eastern Cooperative Oncology Group score of $<2$. One cycle of chemotherapy was administered whilst ventilator support was provided, and the patient's condition eventually improved. However, the patient finally succumbed to respiratory failure at 10 months post-diagnosis.
\end{abstract}

\section{Introduction}

Small cell lung cancer (SCLC) is common in thoracic neoplasms, which currently accounts for $13.6 \%$ of all lung cancers (1), with a high degree of malignancy and rapid tumor progression. SCLC is often diagnosed with widespread metastases at the time of the initial diagnosis. The tumor is sensitive to chemotherapy and radiotherapy, and in the majority of patients, can be controlled in the short-term by chemotherapy. Although many patients are treated with chemotherapy, either alone or in combination with local therapy such as radiation therapy. However, in recent decades, there have no substantial changes in treatment of or improvement in survival from this disease. The 5-year survival of limited stage SCLC is only $20-25 \%$. For extensive stage disease, $<10 \%$ of patients alive at 2 years (1). However, a small proportion of patients in the later stage of SCLC are in a poor physical condition and exhibit disqualifying chemoradiotherapy indications, which makes

Correspondence to: Professor Qiang Li, Department of Chest Surgery, Sichuan Cancer Hospital, 55 Renmin South Road, Chengdu, Sichuan 610041, P.R. China

E-mail: liqiang201320@163.com

Key words: small cell lung cancer, chemotherapy, ventilator them ineligible for chemotherapy treatment. Unfortunately, no molecularly targeted therapy has demonstrated a benefit in SCLC. Therefore, treatment options SCLC are limited only including oral etoposide capsules, pain treatments and other symptomatic treatment. The present study reports the case of a patient with SCLC who was admitted to the Department of Chest Surgery, Sichuan Provincial Cancer Hospital (Chengdu, Sichuan, China) in January 2014, with an Eastern Cooperative Oncology Group (ECOG) (2) score of <2. One cycle of chemotherapy was administered whilst ventilator support was provided, and the patient's condition improved for a time.

\section{Case report}

A 62-year-old female was admitted to the Department of Chest Surgery, Sichuan Provincial Cancer Hospital on January 15, 2014, due to lumbago and backache that had persisted for $>1$ month. Upon admittance, the patient experienced unprovoked lumbago, backache and stomachache, without a cough, sputum, hemoptysis, melena or any other discomfort. The pain was stopped after taking ibuprofen and codeine phosphate tablets. Two weeks later, the patient had to return to hospital since the pain had become subsequently aggravated and could not be relieved by anodyne treatment. The patient reported a history of chronic obstructive pulmonary disease and had been treated with endotracheal intubation and ventilator support in another hospital due to an acute lung infection and type II respiratory failure 1 year previously. A physical examination showed the following: Temperature, $36.3^{\circ} \mathrm{C}$; heart rate, 106 beats/min; respiratory rate, 22 breaths/min; and blood pressure, 114/75 $\mathrm{mmHg}$. There were no signs of superficial lymph nodes on the neck, armpit or groin, but an emphysematous chest, decreased respiratory movement, dullness to percussion at the lower right chest and reduced breath sounds in each lung (particularly in the right lower lobe), without any wheezing, rales or rhonchi, were observed. The patient presented with a normal border of cardiac dullness, a regular cardiac rhythm and no pathological murmurs. The abdomen was flat and soft, while pain was experienced in the mid-upper abdomen under the application of pressure. A hard tumor mass with a diameter $\sim 4 \mathrm{~cm}$ and an irregular shape could be felt. Furthermore, there were no signs of shifting dullness in the abdomen or swelling in the lower extremities.

Subsequent to being hospitalized, the patient's routine blood examination showed the following: Red blood cell 
count, $3.63 \times 10^{12} / 1$; white blood cell count, $8.74 \times 10^{9} / 1$; hemoglobin level, $114 \mathrm{~g} / \mathrm{l}$; and platelet count, $2.13 \times 10^{14} / 1$. Liver function tests revealed the following: Aspartate aminotransferase, $6 \mathrm{U} / \mathrm{l}$; alanine aminotransferase, $13 \mathrm{U} / \mathrm{l}$; serum total bilirubin, $22.5 \mu \mathrm{mol} / 1$; conjugated bilirubin, $4.7 \mu \mathrm{mol} / 1$; and albumin, $37 \mathrm{~g} / \mathrm{l}$. Renal function test results were as follows: Creatinine clearance, $78 \mathrm{ml} / \mathrm{min} / 1.73 \mathrm{~m}^{2}$; creatinine, $47 \mu \mathrm{mol} / \mathrm{l}$; uric acid, $177 \mu \mathrm{mol} / 1$; and blood urea nitrogen, $2.0 \mu \mathrm{mol} / 1$. Other biochemical test results were normal. Computed tomography (CT) scans performed in another hospital showed a space-occupying lesion in the hilus of the right lung, accompanied with pulmonary atelectasis in the lower lobe, medium pleural effusion in the right chest and a space-occupying retroperitoneal lesion. The initial diagnosis considered right-sided lung cancer or lymphoma. A bronchoscopy showed that there was a neoplasm blocking the lateral branch of the middle right lung, with a hyperemic surface; the tissue was soft and bled easily in the process of the biopsy. The biopsy results showed no signs of cancer, but chronic inflammation of the mucous membrane of the lateral branch of the middle right lung. Moderate damage was identified by pulmonary function test. The bones were not involved according to bone scans. The pre-operative blood gas analysis results were as follows: $\mathrm{pH}, 7.49$; partial pressure of carbon dioxide $\left(\mathrm{PCO}_{2}\right), 37.4 \mathrm{mmHg}$; partial pressure of oxygen $\left(\mathrm{PO}_{2}\right), 118.1 \mathrm{mmHg}$; and $\mathrm{HCO}_{3}^{-}, 23.9 \mathrm{mmol} / \mathrm{l}$. The enhanced CT scan of the brain, chest and midsection that was performed in Sichuan Provincial Cancer Hospital on January 15, 2014, revealed a large confluent mass of shadows (the largest at $\sim 5.5 \times 4.5 \mathrm{~cm}$ ) located by the hilus of the right lung and adjacent organs in the mediastinum below the aortic knuckle, as well as an arc liquid density shadow on the dorsal region of the right chest. In addition, multiple space-occupying lesion shadows (the largest at $\sim 6.0 \times 5.2 \mathrm{~cm}$ ) were observed in the retroperitoneal region; some of which were mixed together as one mass, wrapping around the celiac axis, common hepatic artery, splenic artery, and left and right renal arteries, forcing the pancreas to move forward. No craniocerebral space-occupying focus or other evident abnormalities were identified (Fig. 1).

In order to determine an exact diagnosis, the patient underwent a tumor biopsy on the hilus of the right lung via thoracoscopy under general anesthesia on June 20, 2014. Intraoperative findings showed consolidation and atelectasis in the right lobus inferior pulmonis, pale red color and bloody hydrothorax in the right chest ( $\sim 500 \mathrm{ml})$, and a lump of $\sim 6 \mathrm{~cm}$ in diameter at the hilus of the right lung. The post-operative biopsy results indicated small cell carcinoma (Fig. 2): Small cells with little cytoplasm, indistinct cell borders, finely granular nuclear chromatin and absent or inconspicuous nucleolus, nuclear fission were apparent (3). Also, based on the positive tumor immunohistochemical results for cytokeratin, synaptophysin, cluster of differentiation 56, thyroid transcription factor-1, paired box protein Pax-5, with a Ki-67 rate of $\sim 80 \%$, small cell neuroendocrine carcinoma was confirmed.

The post-operative diagnosis showed that the right pulmonary small cell cancer was in an extensive stage, accompanied with abdominal lymph node metastasis (www.nccn.org/professionals/physician_gls/f_guidelines.asp). The patient presented with major discomfort on coughing, sputum excretion and somnolence on January 27, 2014. Emergency blood gas analysis indicated respiratory failure and retention of $\mathrm{CO}_{2}(\mathrm{pH}, 7.27$;
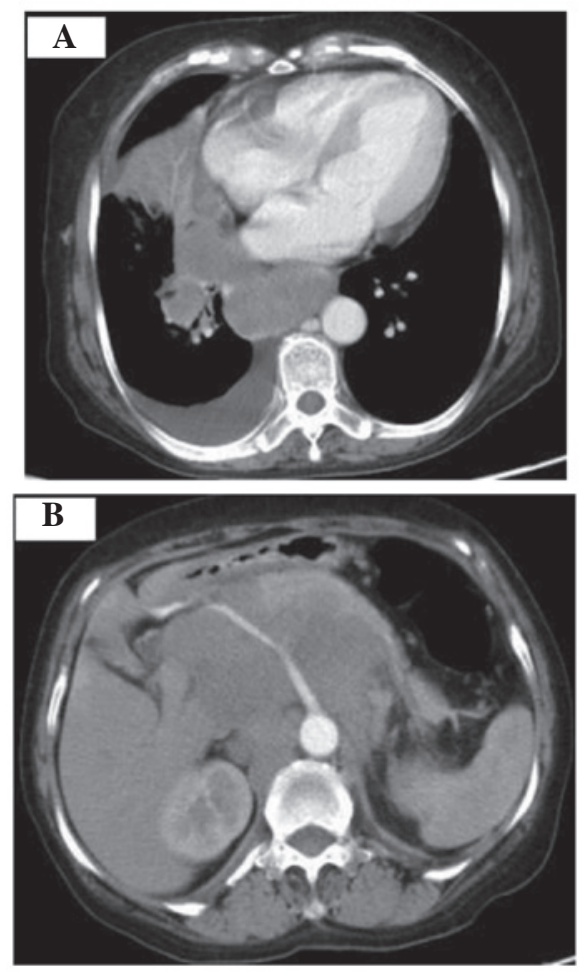

Figure 1. Contrast-enhanced chest and midsection computed tomography demonstrating (A) a large confluent mass shadow (the largest at $\sim 5.5 \times 4.5 \mathrm{~cm}$ ) located by the hilus of the right lung and adjacent organs in the mediastinum below the aortic knuckle, as well as an arc liquid density shadow on the dorsal part of the right chest. (B) Multiple space-occupying lesion shadows (the largest at $6.0 \times 5.2 \mathrm{~cm}$ ) were observed at the retroperitoneal region, some of which were mixed together as one, wrapping around the celiac axis, the common hepatic artery, the splenic artery, and the left and right renal arteries, forcing the pancreas to move forward.

$\mathrm{PCO}_{2}, 116.1 \mathrm{mmHg} ; \mathrm{PO}_{2}, 104.5 \mathrm{mmHg}$; and $\mathrm{HCO}_{3}{ }^{-}, 3.9 \mathrm{mmol} / \mathrm{l}$ ). The patient was then transferred to the Intensive Care Unit (ICU) for respiratory assistance by non-invasive respirator. A blood routine examination showed a white blood cell count of $18.74 \times 10^{9} / 1$, and sputum culture showed growth of Acinetobacter baumannii. The symptoms lessened after 2 days of cefoperazone sodium $(3 \mathrm{~g}$, every $8 \mathrm{~h}$ ) treatment.

However, the somnolence reappeared and the patient presented with confusion on February 4, 2014. A blood gas analysis revealed the following: $\mathrm{pH}, 7.20 ; \mathrm{PCO}_{2}$, $>130 \mathrm{mmHgPO}_{2}, 104 \mathrm{mmHg}$; and $\mathrm{HCO}_{3}^{-}, 29.5 \mathrm{mmol} / \mathrm{l}$. The patient was once again transferred to the ICU for a trachea cannula and respiratory assistance using a respirator. Retention of $\mathrm{CO}_{2}$ decreased 4 days later, but the patient experienced shortness of breath when the respirator was stopped. Use of the respirator long-term would postpone the treatment of the cancer, and aggravation of the cancer would make extubation even more difficult. However, since small cell lung cancer is sensitive to chemotherapy, alveolar recruitment and respiratory function improvements may be a result of a reduced tumor size following effective chemotherapy treatment. After communicating with the patient's family members, chemotherapy was requested. Therefore, the patient received one cycle of chemotherapy (30 mg cis-platinum and $100 \mathrm{mg}$ etoposide on days 1-3) while using a ventilator from February 8-10, 2014. Knowledge of the patient's respiratory failure and dependence 

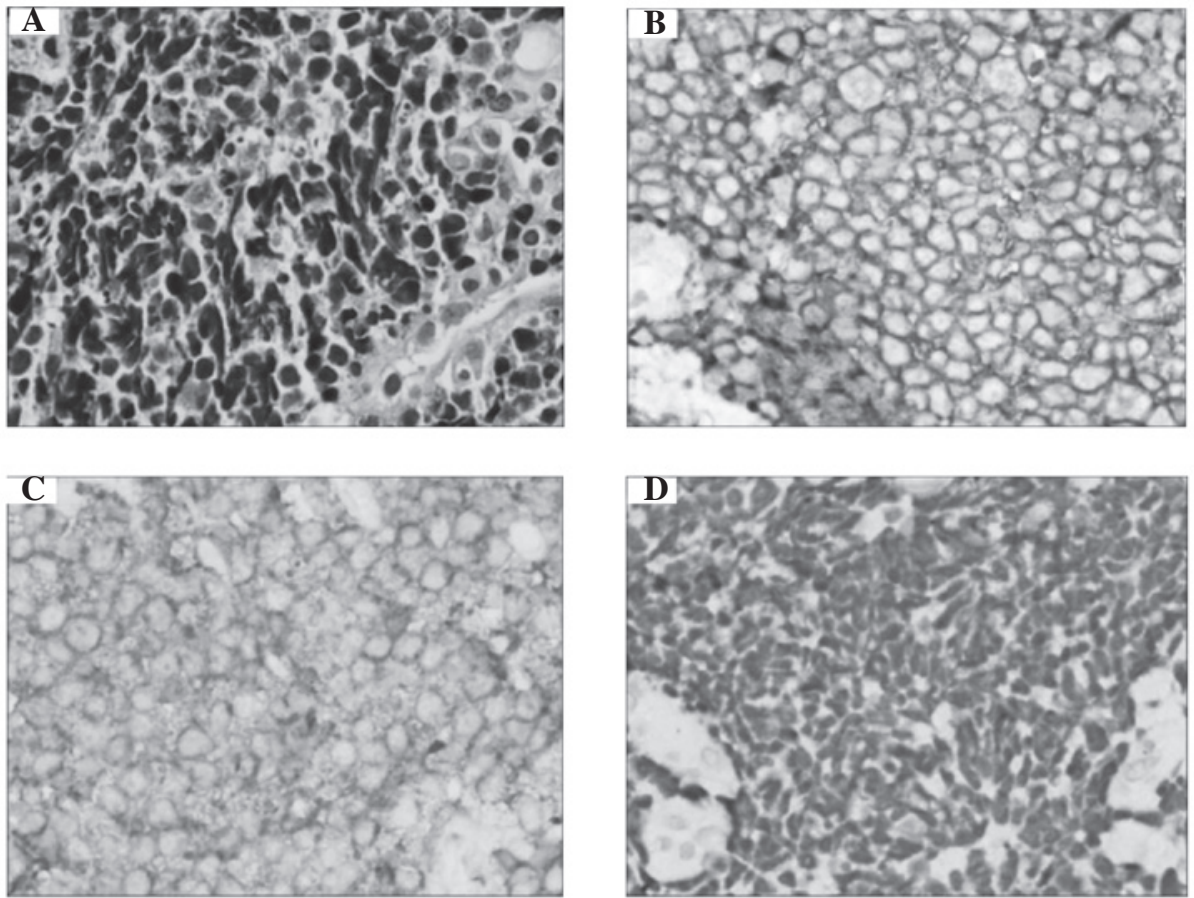

Figure 2. Needle biopsy specimen of the mediastinal mass. (A) Hematoxylin and eosin staining, and (B) neural cell adhesion molecule, (C) synaptophysin and (D) thyroid transcription factor-1 immunostaining. Original magnification, $\mathrm{x} 400$.
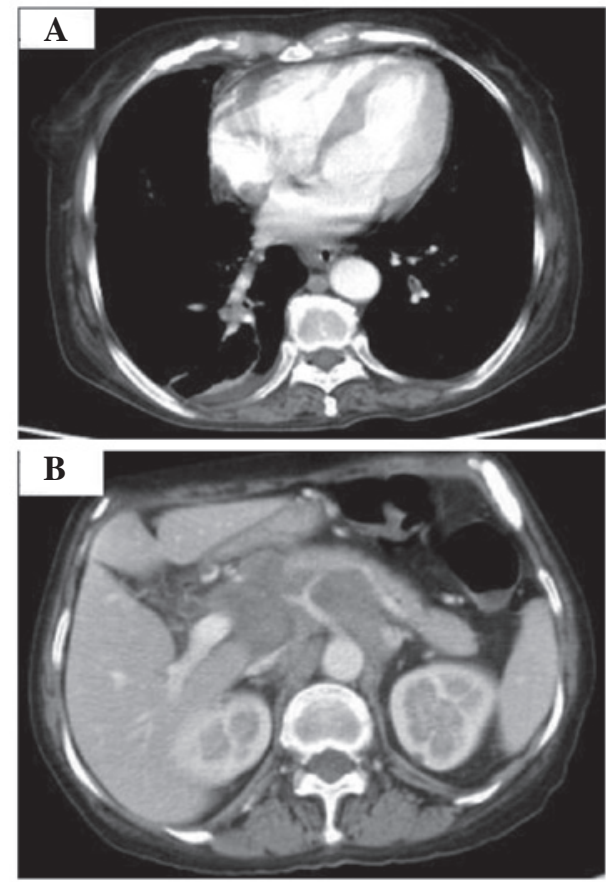

Figure 3. Contrast-enhanced chest and midsection computed tomography at the completion of chemotherapy. The mass located at the hilus of the right lung was smaller than previously $(\sim 3.9 \times 3.1 \mathrm{~cm})$. (A) Pulmonary atelectasis in the lower lobe of the right lung was less severe than previously. (B) The multiple, syncretic and swollen lymph nodes of the hilus of the right lung, abdomen and retroperitoneal area were clearly minimized. The mass in the abdomen was $\sim 4.2 \times 3.1 \mathrm{~cm}$ in size.

on the ventilator meant that intake and output volume, and renal function were under strict monitoring and control during the chemotherapy treatment. In addition, ondansetron $(8 \mathrm{mg}$, every $12 \mathrm{~h}$ ) was used in order to prevent gastrointestinal reactions such as nausea and vomiting. And a gastric tube was used for gastrointestinal decompression. The patient stopped using the ventilator 10 days after the chemotherapy with a successful extubation. Blood gas analysis results were as follows: $\mathrm{pH}, 7.47$; $\mathrm{PCO}_{2}, 61.1 \mathrm{mmHg}$; $\mathrm{PO}_{2}, 91.4 \mathrm{mmHg}$; and $\mathrm{HCO}_{3}{ }^{-}, 43.9 \mathrm{mmol} / \mathrm{l}$. CT scans of the chest and abdominal area on February 24, 2014, showed that the mass located at the hilus of the right lung was smaller than previously $(\sim 3.9 \times 3.1 \mathrm{~cm})$. The pulmonary atelectasis in the lower lobe of the right lung was less severe than prior to the treatment. The multiple, syncretic and swollen lymph nodes of the hilus of the right lung, abdomen and retroperitoneal area were reduced in number and size. The lump in the abdomen was $\sim 4.2 \times 3.1 \mathrm{~cm}$ in size (Fig. 3). The general condition of the patient improved and the ECOG score decreased to 1 . The intake of oral etoposide capsules (100 mg per day) beginning at the second cycle of chemotherapy caused serious myelosuppression, and the patient rejected oral medication half a month after the start of the chemotherapy treatment. Follow-up was performed every 3 months by telephone after the patient was discharged from the hospital. The patient remained alive with an ECOG score of $\sim 1$ until October 2014, but finally succumbed due to respiratory failure in November 2014.

\section{Discussion}

Small cell lung cancer is a type of malignant solid tumor that is extremely sensitive to chemotherapy and radiotherapy, with an objective remission rate of $50-70 \%$ in recent years (4). The first-line treatments for extensive-stage small cell lung cancer are chemotherapy-oriented therapies for the whole body. For patients with a good physical condition [Zubrod-ECOG-World Health Organization (ZPS) score between 0 and 2], strategies 
such as standard-dose etoposide and cisplatin (EP) and carboplatin and etoposide (CE) can be selected $(5,6)$. For patient with a ZPS score between 3 and 4 points, based on the best supportive treatment, the treatment details should be decided with careful evaluations of the advantages and disadvantages according to the comprehensive tumor score, the patient's physiological functions, and the decisions of the patient and their family $(7,8)$.

In the present study, the patient was confirmed with extensive-stage small cell lung cancer and required ventilator support due to respiratory insufficiency prior to chemotherapy. Furthermore, according to the ECOG standard for physical status (ZPS, 5-points method) (5), the patient had a dissatisfactory score of 4 points. According to ZPS staging, the patient was not suitable for chemotherapy. Lastly, no clinical report on implementing chemotherapy under ventilator support exists in this field.

However, for this case, if the tumor could not be controlled, long-term treatment with the ventilator may have resulted in the patient developing a lung infection and even succumbing to systemic failure. In the case of emergencies caused by tumors that are sensitive to chemotherapy, chemotherapy treatment could relieve symptoms and save the patient's life prior to further treatments $(9,10)$. The present patient exhibited normal liver and kidney functions so that chemotherapy could be tolerated regardless of the respiratory insufficiency. Additionally, ventilator support was able to improve the respiratory function. Therefore, it was determined that the patient may tolerate chemotherapy. Additionally, further treatments may have performed if the patient's condition improved after the first cycle of chemotherapy.

The study results showed that the patient responded to the chemotherapy well, with an improved health status (reduction in tumor size and partial response), which reached the goal of a successful treatment. The treatment experiences of this case suggest that such patients who lose chemotherapy indications according to traditional standard remain able to receive chemotherapy and may obtain good results when the tumor is sensitive to chemotherapy and supportive measures are effective.

\section{Acknowledgements}

The authors greatly appreciate the assistance of the staff of the Department of Thoracic Surgery, Sichuan Cancer Hospital, Sichuan University, and thank them for their efforts.

\section{References}

1. Jett JR,Schild SE,Kesler KA and Kalemkerian GP: Treatment of small cell lung cancer: Diagnosis and management of lung cancer, 3rd ed: American College of Chest Physicians evidence-based clinical practice guidelines. Chest 2013: 143, 2013.

2. Oken MM,Creech RH,Tormey DC,et al.Toxicity and response criteria of the Eastern Cooperative Oncology Group. Am J Clin Oncol 5: 649-655, 1982.

3. Meerbeeck JP, Fennell DA and De Ruysscher DK: Small-cell lung cancer. Lancet 378:1741-1755, 2011.

4. Von Pawel J, Schiller JH, Shepherd FA, Fields SZ, Kleisbauer JP, Chrysson NG, Stewart DJ, Clark PI, Palmer MC, Depierre A, et al: Topotecan versus cyclophosphamide, doxorubicin, and vincristine for the treatment of recurrent small-cell lung cancer. J Clin Oncol 17: 658-667, 1999.

5. Buccheri G, Ferrigno D and Tamburini M: Karnofsky and ECOG performance status scoring in lung cancer: A prospective, longitudinal study of 536 patients from a single institution. Eur J Cancer 32A: 1135-1141. 1996.

6. Verger E, Salamero M and Conill C: Can Karnofsky performance status be transformed to the Eastern Cooperative Oncology Group scoring scale and vice versa? Eur J Cancer 28A: 1328-1330, 1992.

7. DeVita VT Jr, Lawrence TS and Rosenberg SA: Devita, Hellman \& Rosenberg's Cancer: Principles and Practice of Oncology. 8th edition. Lippincott Williams and Wilkins, New York, 2008.

8. Rubin P: Clinical Oncology: A Multi-Disciplinary Approach for Physicians and Students. 8th edition. Saunders, London, 2001.

9. Hanna L, Crosby T and Macbeth F (eds): Practical Clinical Oncology. Cambridge University Press, New York, 2008.

10. Jim Cassidy, Donald Bissett and Spence RAJ (eds): Oxford Handbook of Oncology. Oxford University Press, New York, 2002. 